\title{
L'être humain comme matière première
}

L'anatomie du corps humain en tant que matière première et dépôt de pièces de rechange a une longue tradition. De très beaux gobelets ornés d'os de crâne ou des cordes tressées solidement avec des cheveux de femmes sont aujourd'hui des pièces de musée. Ce que l'on sait moins par contre, ce sont les profits que les familles de bourreaux ont retirés de la vente de la graisse des condamnés pendus. A toutes époques, lorsque ce matériel était précieux, les cadavres des champs de bataille ont été utilisés par des spécialistes à des fins artisanales. Même si le cannibalisme gastronomique, dans notre époque éclairée, ne préoccupe plus que des cinéastes et des criminologues, on clora le sujet sur l'équipe de footballeurs atterris en urgence dans les Andes et qui confessèrent devant la presse avoir mangé leurs congénères décédés.

La médecine moderne se sert aujourd'hui de méthodes plus subtiles et garantit un rendement plus varié et plus lucratif. Gênes, ovules, sperme, embryons, mères porteuses, tout est négociable. Des condamnés à mort exécutés fournissent à l'anatomiste allemand Gunther von Hagens de quoi créer des atlas virtuels ou des formes humaines plastinifiées. Dans la zone grise du marché global, des entreprise privées ou étatiques saisissent les chances qu'offre un tourisme médical croissant. Les obstacles juridiques, comme la nouvelle loi helvétique sur la transplantation, sont relativement aisés à contourner pour le riche consommateur. Les risques peuvent être plus grands, certes, mais le professionnalisme n'existe pas que dans notre pays. Le trafic d'organes est une branche en pleine expansion, stimulée par la disparité entre riches et pauvres, par l'illusion d'une faisabilité sans limite et le souhait d'une absolue longévité. Sommes-nous aussi civilisés que nous voudrions le paraître? Des motifs semblables ne se révèlent-ils pas lorsque l'on «consomme» un organe en se l'appropriant par une opération ou que l'on consomme un repas? Le cadre culturel ne varie que peu. Une énergie symbolique, spirituelle ou matérielle, se dirige du donateur, mort ou vivant, vers celui qui reçoit. Autrefois, ce pouvait être le courage d'un ennemi vaincu ou la force des ancêtres. Aujourd'hui, personne n'a besoin de boire du sang comme Dracula pour prolonger sa vie. En s'habituant à l'idée des organes étrangers, on n'aura plus besoin d'un soutien psychologique pour briser le tabou. Le cœur est un muscle creux et n'est plus, depuis longtemps, l'habitat d'une âme pleine de mauvais augures. Le foie, les poumons et l'intestin grêle sont, quant à eux, moins chargés d'émotions et la voie est même déjà tracée vers la transplantation chirurgicale d'un visage étranger. Les lois et les ordonnances gèrent les contraintes qui résultent du fait accompli que représente la technique médicale. Peut-être aurait-on pu renoncer à un premier pas dans cette voie, mais le second est inéluctable. On peut imaginer qu'il n'y ait pendant longtemps pas d'alternative à des organes provenant d'autres humains, mais que les réactions de rejet deviennent plus faciles à contrôler dans le futur. La demande massive suivra alors la logique du marché. La qualité est demandée, pas la morale. Que les reins continuent de provenir de Moldavie ou de Chine ne joue qu'un rôle secondaire. Aujourd'hui déjà, plus personne dans un congrès européen ne se demande comment ses confrères chinois ont acquis leur dextérité en transplantation. Même si l'on sait que les détenus sont des banques d'organes et que cela soulève des protestations mondiales, cela ne gêne en aucune manière le commerce avec la Chine. Poursuivre en justice des médecins de pays puissants est quasiment impossible. Ce fut déjà le cas en Union soviétique, lorsque des confrères traitaient des opposants comme s'ils étaient des malades psychotiques. Le malaise est profond, parce que nous sommes ambivalents. Le vieux cannibale en nous et la peur qu'il nous inspire n'ont disparu qu'en apparence. Nombre de médecins aux intentions les plus louables manquent de ce que le philosophe Hans Jonas décrit dans son ouvrage comme étant «le principe de responsabilité», à savoir la clairvoyance de l'imagination et la délicatesse des sentiments. Devant l'illusionnaire espoir de «guérir» ou de trouver le salut, il reconnaît dans la crainte même le premier devoir d'une éthique de la responsabilité historique: «le frisson de peur que l'on éprouve à la pensée de ce que l'être humain pourrait devenir et qui nous fait reculer lorsque cette possibilité nous dévisage fixement depuis un avenir anticipé». Le précurseur de la «durabilité» pensait sans aucun doute par là à l'exploitation technique de tous les domaines scientifiques. Peutêtre que le peu de succès que remporte le don d'organes auprès de notre population émane en fait de cette peur latente. Ce serait un premier pas vers un autre avenir. 